\title{
Évaluation scientifique et tarification des dispositifs médicaux et des actes associés en France
}

\author{
Martine Gilard ${ }^{1}$, Frédérique Debroucker ${ }^{2}$, Claude Dubray ${ }^{3}$ et les participants à la table ronde $N^{\circ} 1$ de Giens \\ XXVIII : Yves Allioux ${ }^{4}$, Eliane Aper ${ }^{5}$, Valérie Barat-Leonhardt ${ }^{6}$, Michèle Brami ${ }^{7}$, Cédric Carbonneil ${ }^{8}$, \\ Emmanuel Chartier-Kastler ${ }^{9}$, Claire Coqueblin ${ }^{10}$, Sandrine Fare ${ }^{11}$, Isabelle Giri ${ }^{12}$, Jean-Marie Goehrs ${ }^{13}$, \\ Karine Levesque ${ }^{14}$, Philippe Maugendre ${ }^{15}$, François Parquin ${ }^{16}$, Jean-Patrick Sales ${ }^{17}$ et Karine Szwarcensztein ${ }^{18 \dagger}$
}

1 Centre Hospitalier Universitaire La Cavale Blanche, Brest, France

2 Medtronic France, Boulogne-Billancourt, France

3 Centre de Pharmacologie Clinique, Clermont-Ferrand, France

4 CNAMTS, Paris, France

5 General Electric, Vélizy, France

6 GlaxoSmithKline, Marly-le-Roi, France

7 Agence Technique de l'Information sur l'Hospitalisation, Paris, France

8 Direction générale de l'Offre de Soins, Paris, France

9 Hôpital Pitié-Salpêtrière, Paris, France

10 DGCIS, Ivry, France

11 Direction de la Sécurité sociale, Paris, France

12 Access Conseil, Paris, France

13 JMGoehrs Partners, Versailles, France

14 Abbott Vascular, Rungis, France

15 Sanofi Aventis, Paris, France

16 Hôpital Foch, Boulogne-Billancourt, France

17 Haute Autorité de Santé, Saint-Denis La Plaine, France

18 Johnson \& Johnson, Issy-les-Moulineaux, France

Texte reçu le 15 mars 2013 ; accepté le 7 mai 2013

\section{Mots clés :}

acte ;

évaluation ;

tarification

prise en charge ;

remboursement dispositif médical ;

\begin{abstract}
Résumé - Le dispositif médical se caractérise par une grande hétérogénéité (de l'abaisse langue à l'équipement d'imagerie, en passant par le dispositif implantable ou invasif), un cycle de vie court des produits avec des innovations incrémentales récurrentes (de 18 mois à 5 ans) et un caractère opérateur dépendant : le plus souvent l'utilisateur clinicien doit réaliser un acte de pose (prothèse de hanche, stimulateur cardiaque), un acte thérapeutique qui utilise un dispositif invasif non implantable (sonde d'ablation d'un foyer arythmique, ballonnet d'angioplastie, système de spondyloplastie par extension) voire un acte de suivi des dispositifs actifs implantés (suivi au long court des patients bénéficiant d'un défibrillateur cardiaque, ou des malades parkinsoniens implantés d'un système de stimulation cérébrale profonde).

La table ronde a souhaité se concentrer sur les procédures d'évaluation scientifique des dispositifs médicaux et des actes associés en vue de leur tarification et financement par l'Assurance maladie. Elle a ainsi posé pour postulat que le corpus de données disponibles était suffisant et compatible avec une évaluation scientifique du bénéfice clinique. Les études post-inscription, qui participent à la continuité de l'évaluation des dispositifs médicaux, n'ont pas été abordées. En outre, elle a choisi de centrer ses réflexions sur les dispositifs utilisés dans les établissements de santé (ES), les plus représentatifs de l'association entre dispositifs et actes médicaux techniques.

Le contexte des multiples procédures réglementaires, permettant la prise en charge des dispositifs médicaux et des actes, est rappelé. Les problématiques liées plus spécifiquement aux actes eux-mêmes, aux dispositifs médicaux non implantés et aux dispositifs médicaux innovants sont ensuite développées. Dans chacune des trois situations, les propositions et les points de discussion de la table ronde sont présentés.
\end{abstract}

Abréviations : voir en fin d'article. 


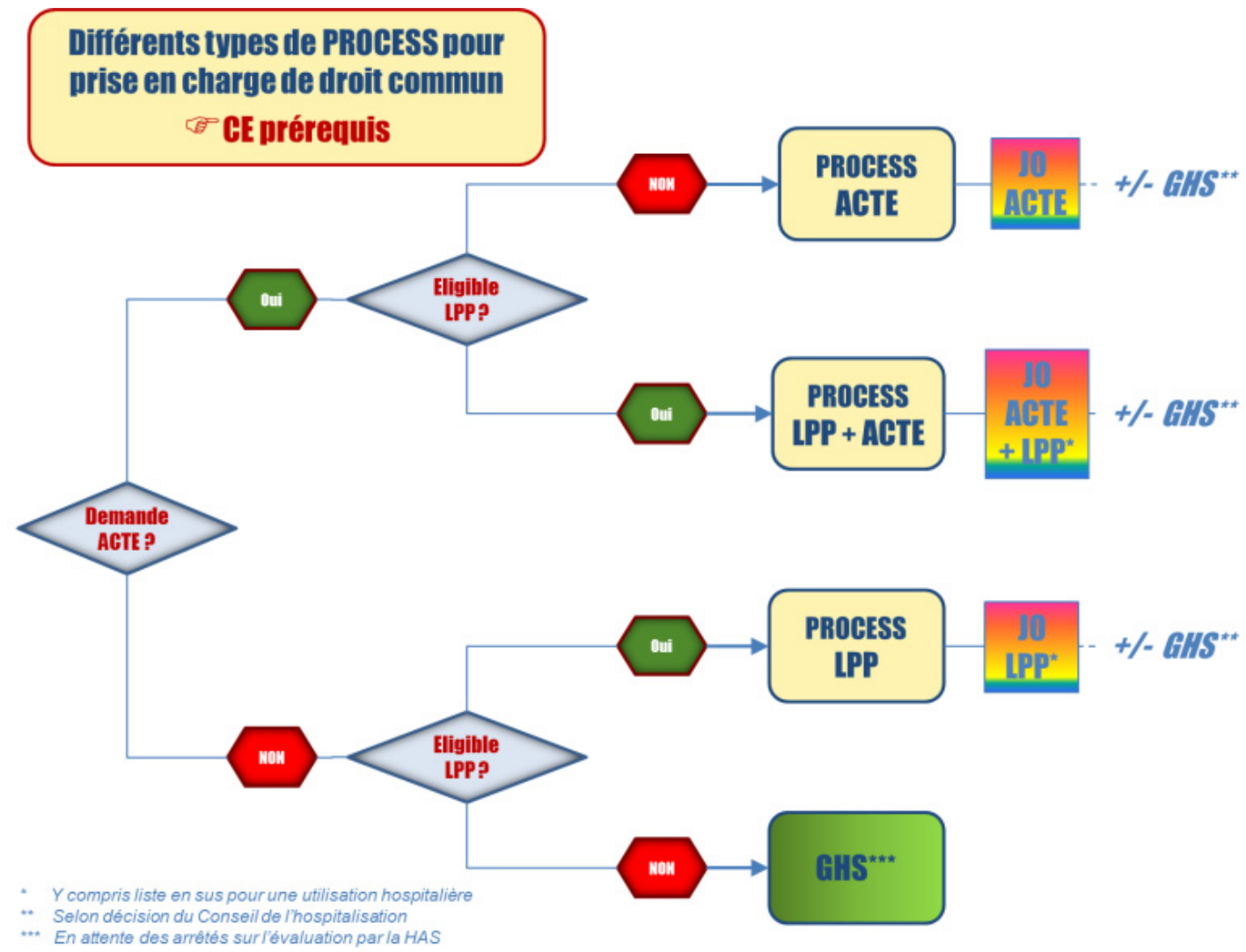

Fig. 1. Procédures d'évaluation et de tarification en vue d'une prise en charge de droit commun des dispositifs médicaux et des actes associés. GHS : goupe homogène de séjour ; JO : journal officiel ; LPP : liste des produits et prestations remboursables.

\section{Contexte}

En France, les procédures d'évaluation et de tarification des dispositifs médicaux et des actes associés sont multiples et complexes (figure 1). Elles reposent sur deux questions préalables :

- Est-il nécessaire de demander la création d'un acte technique qui ne serait pas encore référencé dans les nomenclatures (classification commune des actes médicaux [CCAM], nomenclature générales des actes professionnels [NGAP], nomenclature des actes de biologie médicale [NABM]) pour que le dispositif médical soit utilisé ?

Si tel est le cas, la Haute autorité de santé (HAS) peut être saisie pour réaliser l'évaluation scientifique de l'acte soit par la ou les sociétés savantes concernées, via la soumission d'un formulaire de quelques pages, soit par le Ministère de la santé, soit par l'Union nationale des caisses d'Assurance maladie (UNCAM). Dans le premier cas, l'inscription du dossier au programme de travail de la HAS n'est pas automatique, comme on le verra ultérieurement.

La tarification de l'acte est ensuite réalisée à partir de l'avis consultatif du Collège de la HAS par le pôle nomenclature de l'UNCAM après négociation avec les professionnels de santé.
- Le dispositif médical remplit-il les conditions d'éligibilité à la liste des produits et prestations remboursables $(L P P)$ définie par l'article L.165-1 du Code de la sécurité sociale (annexe 1) ?[1]

Dans ce cas, soit il existe une ligne générique préalable permettant l'inscription du dispositif sur la LPP par l'industriel, soit ce dernier doit déposer un dossier complet de demande d'inscription sur la LPP « sous nom de marque » auprès de la commission spécialisée de la HAS, la Commission nationale d'évaluation des dispositifs médicaux et des technologies de santé (CNEDiMTS) et du Comité économique des produits de santé (CEPS) [annexe 2].

La CNEDiMTS réalise l'évaluation scientifique du dispositif à partir des données disponibles. La tarification est effectuée par le CEPS à partir de l'avis consultatif de la CNEDiMTS de la HAS, après négociation avec l'industriel.

S'il s'agit d'un dispositif médical implantable utilisé en établissement de santé, il peut être alors inscrit à la fois sur la LPP et sur la liste « en sus », permettant son financement « en sus » des forfaits hospitaliers (groupe homogène de séjour ou GHS ; le GHS est une prestation hospitalière qui rémunère de façon forfaitaire un séjour hospitalier). Cette inscription n'est possible qu'après un avis positif du Conseil de l'hospitalisation qui évaluera l'hétérogénéité induite 
dans la prise en charge du séjour hospitalier, son caractère onéreux et les modalités de son utilisation (annexe 2).

L'ensemble de la procédure d'évaluation et de tarification est encadrée par un délai réglementaire de 180 jours. En pratique, le délai total moyen pour une première inscription d'un dispositif médical à la LPP est d'environ 9 mois, dont l'évaluation par la CNEDIMTS a représenté 85 jours en moyenne en 2011.

Si la réponse est « oui » aux deux questions précédentes, alors une procédure double doit être mise en place. Le dépôt du dossier de l'industriel va déclencher une autosaisine de la HAS qui basera l'évaluation de l'acte sur ce même dossier avec éventuellement le recours à un groupe de travail ad hoc. L'évaluation de l' acte est ainsi « accrochée » à celle du dispositif.

Enfin, pour les dispositifs médicaux qui ne sont pas éligibles à la LPP et qui sont donc inclus dans la tarification intra GHS, l'évaluation scientifique de la thérapie peut avoir lieu par le biais de l'acte s'il n'en existe au préalable pas ou, s'il y a lieu, via la prochaine évaluation des catégories de dispositifs «intra-GHS » définie par l'article L.165-11 du CSS ${ }^{\text {[2] }}$ crée par la Loi relative au renforcement de la sécurité sanitaire du médicament et des produits de santé du 21 décembre 2011. Le financement du dispositif médical est alors assuré par les forfaits hospitaliers (GHS).

Toutefois un industriel a toujours la possibilité de soumettre un dossier en vue d'une inscription à la LPP pour un produit dont la catégorie correspondante a fait l'objet d'une intégration dans les GHS.

\section{Acte}

Le tableau I reprend les différentes étapes de l'évaluation et de la tarification d'un acte.

Ce tableau montre la complexité et l'étendue de l'évaluation scientifique réalisée par la HAS d'une part, à qui il revient d'analyser la littérature et de rechercher un consensus scientifique auprès des différents professionnels de santé concernés. Il souligne d'autre part la multiplicité des acteurs engagés autour de la hiérarchisation et de la tarification des actes par l'Union nationale des caisses d'Assurance maladie (UNCAM), avec des étapes nombreuses et peu lisibles. De plus, il existe peu de délais réglementaires encadrant ces procédures.

Enfin, dans l'ensemble du processus, l'industriel concerné par la demande ne dispose d' aucun rôle et sa participation n'est pas officiellement reconnue.

L'état des lieux est en conséquence très mitigé et fonction des situations rencontrées.

Concernant l'évaluation par la HAS :

- en cas de saisine par l'UNCAM, la procédure est structurée et les délais, encadrés, restent prévisibles ;

- lorsque l'acte est accroché à un dispositif LPP, son évaluation est réalisée au minimum sur la base du dossier du fabricant.
La procédure est là aussi structurée et les délais sont encadrés par ceux liés à l'évaluation du dispositif ;

- le cas des biomarqueurs compagnons (c'est-à-dire dans le cas d'un biomarqueur dont la détermination par le biais d'un acte diagnostic est nécessaire (stipulé dans l'autorisation de mise sur le marché [AMM]) à la prescription d'un médicament) s'avère plus complexe. En effet, lorsqu'un dossier de demande d'évaluation (note d'intérêt thérapeutique) est déposé auprès de la HAS pour une thérapie ciblée, l'information relative au biomarqueur/test diagnostic compagnon est remontée à la HAS via ce dossier mais cela ne permet pas, à ce jour, de déclencher l'évaluation de l'acte diagnostic associé. L'évaluation est compliquée par le fait que deux objets de nature différente (le médicament et l'acte de diagnostic) doivent être évalués par des instances et selon des procédures différentes. De plus, les industriels sont souvent différents pour le médicament et le test diagnostic et les données exigibles pour ce dernier n'ont pas encore été clairement définies à ce jour. En effet, l'évaluation ne concerne pas uniquement les performances intrinsèques du test mais aussi l'impact sur la stratégie thérapeutique et les autres paramètres relatifs au prélèvement. En oncologie, d'autres acteurs, tels que l'Institut national du cancer (INCA) peuvent dans un premier temps réaliser ces tests afin que ces médicaments ciblés en oncologie soient accessibles via les plateformes de biologie moléculaire pour l'ensemble des patients sur le territoire. L'INCa disposant d'un contingent de programmes hospitaliers de recherche clinique (PHRC) et de programmes de soutien aux techniques innovantes et coûteuses (PSTIC), une intégration plateformes INCa/STIC serait particulièrement intéressante pour apporter des données en soutien de l'évaluation des thérapies ciblées et de leurs tests compagnons ;

- enfin, l'évaluation de l'acte « seul »qu'il soit associé ou non à un dispositif non éligible à la LPP, dépend de la capacité de la HAS à intégrer l'évaluation dans son programme de travail. Ainsi, aucun acte proposé par une ou des société(s) savante(s) n'a été évalué dans ce cadre en 2012. En 2013, sur les 54 demandes déposées par les sociétés savantes (dont moins de la moitié seulement réfère effectivement à l'évaluation d'un acte technique) seuls 4 actes (au plus) pourront être évalués et deux feront l'objet d'analyse détaillée en vue de mieux étayer une demande ultérieure.

Concernant la hiérarchisation par l'UNCAM :

- les étapes, quoique structurées, sont très nombreuses. Elles ne sont pas encadrées par des délais réglementaires;

- la multiplicité des acteurs complexifie cette phase de hiérarchisation ;

- au total, le risque de blocage de la procédure est important. 
Tableau I. Évaluation et tarification d'un acte.

\begin{tabular}{|c|c|c|c|}
\hline Étape & Acteurs & Actions & Délai \\
\hline \multirow{5}{*}{ Saisine de la HAS } & Sociétés savantes & Formulaire de quelques pages & \multirow{3}{*}{$\begin{array}{l}\text { Évaluation par la HAS en fonction de } \\
\text { l'inscription à son programme de tra- } \\
\text { vail }\end{array}$} \\
\hline & Ministère & Lettre de saisine & \\
\hline & Inca & Par l'intermédiaire du ministère & \\
\hline & UNCAM & Lettre de saisine & 180 jours renouvelables une fois \\
\hline & Autosaisine & $\begin{array}{l}\text { En cas d' « accrochage » de l'acte à un } \\
\text { dispositif }\end{array}$ & $\begin{array}{l}\text { Lié au délai d'évaluation du dispositif } \\
\text { (prolongé en cas de groupe de travail) }\end{array}$ \\
\hline \multirow[t]{4}{*}{$\begin{array}{l}\text { Évaluation scientifique } \\
\text { par la HAS }\end{array}$} & $\begin{array}{l}\text { SEAP/HAS } \\
\text { (Service d'évaluation des actes) }\end{array}$ & $\begin{array}{l}\text { 1/ Cadrage } \\
\text { 2/ Analyse critique de la littérature } \\
\text { 3/ Recueil de la position argumentée des } \\
\text { experts (GT(s), GL, audition, enquête) } \\
\text { Circuit plus court dans le cas d'un acte } \\
\text { «accroché » à un DM }\end{array}$ & \multirow{4}{*}{$\begin{array}{l}\text { Hormis la saisine de l'UNCAM, il } \\
\text { n'existe pas d'obligation réglementaire } \\
\text { pour la HAS à évaluer les actes qui lui } \\
\text { sont soumis. } \\
\text { En moyenne, l'évaluation d'un acte } \\
\text { nécessite entre } 10 \text { et } 14 \text { mois après } \\
\text { démarrage des travaux. }\end{array}$} \\
\hline & CNEDIMTS & $\begin{array}{l}\text { 4/ Examen du rapport et des conclusions } \\
\text { et préparation de l'avis }\end{array}$ & \\
\hline & Collège de la HAS & 5/ Validation du rapport et de l'avis & \\
\hline & SEAP/ HAS & 6/ Diffusion (publication) & \\
\hline \multirow{4}{*}{$\begin{array}{l}\text { Hiérarchisation } \\
\text { par I'UNCAM }\end{array}$} & $\begin{array}{l}\text { Dact (Direction des actes), Sociétés } \\
\text { savantes, ATIH }\end{array}$ & 1/Proposition de libellés & \multirow{4}{*}{ Aucun délai défini } \\
\hline & Panel d'experts et Dact & 2/ Hiérarchisation & \\
\hline & Instance de cohérence & 3/ Validation & \\
\hline & $\begin{array}{l}\text { CHAP (Commission de hiérarchi- } \\
\text { sation des actes et prestations) }\end{array}$ & 4/ Vote de la hiérarchisation & \\
\hline $\begin{array}{l}\text { Évaluation économique par } \\
\text { I'UNCAM }\end{array}$ & UNCAM & $\begin{array}{l}\text { 1/ Tarification } \\
\text { 2/ Évaluation de l'opportunité d'inscrire } \\
\text { 3/ Conditions d'inscription }\end{array}$ & Aucun délai défini \\
\hline \multirow{2}{*}{$\begin{array}{l}\text { Information et consultation } \\
\text { par I'UNCAM }\end{array}$} & UNCAM & $\begin{array}{l}\text { Lettre d'intention pour Ministre, UNPS, } \\
\text { Syndicats concernés, Syndicats des E.S. }\end{array}$ & Information simple \\
\hline & UNOCAM & Consultation & $\begin{array}{l}\text { Délai de } 6 \text { mois ou } 21 \text { jours si ASA I ou } \\
\text { ASA II }\end{array}$ \\
\hline Inscription par l'UNCAM & Collège des directeurs & Décision UNCAM & Aucun délai défini \\
\hline Approbation par le Ministre & Ministre & $\begin{array}{l}\text { Refus possible à partir de la lettre } \\
\text { d'intention et de l'impact financier }\end{array}$ & $\begin{array}{l}\text { Délai de non opposition de } 45 \text { jours } \\
\text { ou } 15 \text { jours si ASA I ou II }\end{array}$ \\
\hline Publication au JO & UNCAM & Publication selon décision UNCAM & Délai selon décision UNCAM \\
\hline
\end{tabular}

ASA : amélioration du service rendu ; ATIH : Agence technique de l'information sur l'hospitalisation ; CHAP : Commission de hiérarchisation des actes et prestations ; DM : dispositif médical ; ES : établissements de santé ; GL : groupe de lecture ; GT : groupe de travail ; HAS : Haute autorité de santé ; SEAP : Service d'évaluation des actes et prestations ; UNCAM : Union nationale des caisses d'Assurance maladie ; UNPS : Union nationale des professionnels de santé ; UNOCAM : Union nationale des organismes d'Assurance maladie complémentaires.

Concernant l'évaluation économique et la tarification par l'UNCAM :

- l'opportunité d'inscription après tarification est définie sur des critères d'impact économique non publics ;

- la décision finale par le Collège des directeurs de l'UNCAM n'est pas publiée et il n'existe pas de procédure contradictoire. In fine, bien que la possibilité d'accrocher l'acte aux dispositifs médicaux LPP ait effectivement permis d' aboutir à la prise en charge de quelques actes associés à des dispositifs d'ASA élevés dans des délais cohérents, dans les autres situations, ces procédures peuvent prendre plusieurs années, voire ne pas aboutir.

Les propositions consensuelles de la table ronde autour de l'évaluation et de la tarification des actes sont les suivantes :

- mieux définir le cahier des charges du dossier acte « seul » et dispositif hors LPP pour documenter et argumenter la demande d'évaluation auprès de la HAS ; 
- clarifier la doctrine d'évaluation spécifique à l'acte en vue de l'inscription (HAS) ;

- légitimer l'apport de l'industriel dans la préparation du dossier de demande de création d'acte et assurer la transparence des échanges entre industriel(s) et organismes professionnels (sociétés savantes) ;

- informer les organismes professionnels sur les procédures d'évaluation et de tarification ;

- engager de plus en plus un processus collaboratif via les Collèges des organisations professionnelles;

- fluidifier et intégrer l'ensemble des procédures ;

- augmenter la capacité à faire de la HAS.

Les points de discussion qui n'ont pas été définitivement actés par les membres de la table ronde sont décrits ci-après :

- réaliser un transfert de la charge d'évaluation vers le dispositif médical afin d'optimiser l'évaluation en maintenant la qualité ;

- autoriser un dépôt commun industriel/société savante ;

- favoriser l'apport d'experts pratiquant l'acte, que ce soit lors de l'évaluation scientifique ou de la hiérarchisation. Il conviendrait alors de gérer les potentiels liens d'intérêt, qu'ils soient positifs ou négatifs ;

- clarifier les critères de l'UNCAM déterminant l'inscription ou non d'un acte.

\section{Dispositif médical non implanté " onéreux »}

Les dispositifs médicaux non implantés onéreux forment une catégorie particulière : il s'agit de dispositifs médicaux utilisés au moment de l'acte thérapeutique, invasifs, qui ne restent pas dans le corps humain un temps suffisant - 30 jours - pour être éligibles au titre III de la LPP. En effet, les conditions générales de la LPP stipulent qu'un dispositif ne peut être inscrit sur le titre III que s'il remplit les conditions suivantes :

1. Implantation en totalité dans le corps humain.

2. Pose uniquement par un médecin.

3. Durée d'implantation supérieure à 30 jours.

4. Être l'objectif principal de l'intervention.

Ainsi, le dispositif médical non implanté, par son coût ou l'utilisation qui en est faite, peut induire une hétérogénéité dans le forfait hospitalier de prise en charge. On peut citer :

- les sondes d'ablation des foyers de fibrillation atriale ;

- les ballonnets intra-utérins pour thermo-coagulation de l'endomètre ;

- les ballonnets à élution de principe actif utilisés au cours d'angiographie coronarienne ;

- les ballonnets à élution de principe actif utilisés dans le traitement des artériopathies obstructives des membres inférieurs ;
- la mesure du flux coronaire résiduel (fractional flow reserve [FFR]) lors d'un cathétérisme cardiaque.

Ces dispositifs n'étant pas éligibles à la LPP, l'évaluation de leur bénéfice thérapeutique par la HAS, légitimement nécessaire à toute potentielle adaptation du financement, ne peut passer que par l'évaluation de l'acte thérapeutique associé, lorsqu'il n'existe pas au préalable. En revanche, si un acte approprié préexiste, ces dispositifs n'ont jusqu'à présent pas accès à l'évaluation par la HAS.

Concernant leur financement, ces dispositifs médicaux sont pris en charge via les forfaits hospitaliers, ou GHS, définis par la tarification à l'activité (T2A). Cette dernière a pour objectif d'équilibrer un éventail des cas : chaque séjour est groupé dans un groupe homogène de malades (GHM) et la $\mathrm{T} 2 \mathrm{~A}$ rémunère l'établissement selon un tarif, le GHS, calculé à partir de la moyenne de la dépense engagée par les établissements participant à l'Étude nationale des coûts (ENCC [étude financière permettant de calculer les coûts réels moyens pour l'établissement de santé par GHS à partir de la comptabilité analytique des centres participants]), pour les malades dont les séjours sont groupés dans le même groupe homogène de malade. Le GHS final est ajusté en tenant compte des coûts retrouvés dans l'ENCC mais aussi des autres financements des établissements de santé.

Les éléments risquant d'induire une distorsion dans l'homogénéité du GHM font l'objet d'un financement en sus du GHS : séjours particulièrement longs ou courts, réanimation, soins intensifs, molécules onéreuses et dispositifs médicaux implantables. En revanche, le cas des dispositifs non implantés onéreux, peu nombreux lors de la création de la T2A, n'a pas été prévu.

En outre, il n'existe pas de procédure formalisée permettant une adaptation du GHS suite à l'évaluation de ces dispositifs et des actes associés par la HAS. Toutefois, lors de la mise en place de la T2A, on a procédé de façon très rare, à la déclinaison d'un même groupe homogène de malade en 2 , voire plusieurs, forfaits hospitaliers (GHS), pour prendre en compte un surcoût lié à l'utilisation d'un « consommable » onéreux. Cette solution reste théoriquement possible quoique difficile à mettre en œuvre, et reste dépendante de l'existence d'un «marqueur» de l'utilisation du consommable onéreux : code CCAM par exemple. Il faut noter que le mode de calcul des GHS va rendre compte de l'hétérogénéité induite par ces dispositifs médicaux non implantés onéreux, mais avec un décalage de 2 ans. De plus, l'impact peut rester très peu visible lorsque l'utilisation du dispositif n'est pas systématique, ce qui se produit souvent lorsque le financement reste insuffisant.

Sur ce sujet des dispositifs médicaux non implantés onéreux, appelés aussi couramment « consommables onéreux », de plus en plus nombreux, seuls des points de discussion ont été définis par la table ronde :

- dimensionner le GHS à partir de l'acte associé au dispositif lorsqu'il existe. Cette solution doit rester exceptionnelle, et les conditions de son application restent à définir (intérêt thérapeutique élevé, impact financier sur le GHS ?) ; 
- si la création d'un acte spécifique n'est pas nécessaire :

- rendre possible l'inscription sur la liste en sus d'un DM non éligible à la LPP ou bien revoir les critères d'éligibilité à la LPP (ex: la durée d'implantation de 30 jours),

- demander une évaluation intra-GHS tel que définie par l'article L. 165-11 du CSS ${ }^{[2]}$ créé par la Loi Bertrand fin 2011, pour ouvrir la discussion sur le financement en cas d'intérêt thérapeutique élevé.

Dans tous les cas, il est important de souligner que la procédure d'adaptation du financement n'est pas définie.

\section{Dispositif médical innovant}

Les dispositifs médicaux innovants et actes associés peuvent, dans certains cas, bénéficier du PHRC et du PSTIC. Ces programmes sont gérés par la Direction générale de l'offre de soins (DGOS). Ils ne sont ni nécessaires, ni suffisants pour justifier d'une prise en charge par la collectivité. Ils représentent toutefois une aide mise à la disposition des centres français permettant de réaliser une étude particulière, dans le contexte français, qui viendra compléter les données cliniques disponibles par ailleurs. C'est bien l'ensemble de ces données qui permettra d'engager une évaluation pour la prise en charge de la technologie de santé.

Le PHRC permet, notamment, d'évaluer la sécurité et l'efficacité clinique de la technique via le financement d'une recherche comparative. Le PSTIC se situe en aval du PHRC ou des études équivalentes. Il a pour objectif de valider l'utilité clinique et médico-économique d'une technique en vue d'une prise en charge par la collectivité. Il se situe en amont de l'évaluation par la HAS. C'est pour cette raison que la DGOS et la HAS ont décidé qu' aucune évaluation ne pouvait être engagée par cette dernière sur une technologie de santé pour laquelle un PHRC ou un PSTIC est en cours. Les procédures de soumission et de sélection des projets PHRC et PSTIC ont été grandement clarifiées et structurées en 2011.

Cependant, la soumission à ces programmes revenant aux équipes hospitalières françaises, il arrive que les études qu'elles proposent ne soient pas en concordance avec les programmes de recherche clinique internationale développés par les industriels ou même qu'elles soient pour partie redondantes. Dans certains cas, l'implémentation d'un PHRC ou un PSTIC peut ainsi retarder sensiblement l'évaluation d'une technologie de santé par la HAS, alors même que le corpus de données nécessaire à cette évaluation est disponible par ailleurs.

En aval de l'évaluation par la HAS, plusieurs dispositions réglementaires permettent de prendre en charge et d'encadrer les techniques innovantes.

Le «forfait innovation », créé par la loi «Hôpital, patients, santé, territoire » (HPST) en 2009 (Article L.165-1-1 du CSS), ${ }^{[3]}$ consiste en une prise en charge dérogatoire et temporaire d'une technologie innovante. Il concerne les produits, prestations et/ou actes innovants pour lesquels la HAS a considéré que les données n'étaient pas suffisantes pour proposer une prise en charge par la collectivité. Cette prise en charge est dérogatoire car conditionnée à la réalisation d'une étude. Cette étude doit permettre de répondre aux incertitudes soulevées par l'évaluation par la HAS. Il s'agit donc avant tout d'une étude clinique et éventuellement médico-économique en fonction de la question posée.

La HAS a proposé l'application du forfait innovation à plusieurs techniques fin 2010. Les dossiers retenus suivent leur cours depuis 2 ans, mais pour le moment aucun n'a abouti.

L'article L.1151-1 du CSP ${ }^{[4]}$ permet quant à lui d'encadrer la réalisation ou la pratique d'une technique innovante. Cette disposition est applicable uniquement en aval d'une évaluation HAS en cas de service attendu suffisant, et concerne les actes, procédés, techniques, méthodes à visée diagnostiques ou thérapeutiques et les dispositifs médicaux. Elle est utilisée lorsqu'un encadrement est justifié pour raison de santé publique ou lorsque la technique est susceptible d'entraîner des dépenses injustifiées. Cet encadrement définit des règles de bonnes pratiques, des conditions techniques de fonctionnement, formation et qualification des professionnels de santé impliqués. L'utilisation de la technique peut être limitée à certains établissements de santé. L'article 1151-1 du $\operatorname{CSP}^{[4]}$ a été appliqué avec succès aux valves cardiaques aortiques percutanées.

Enfin, lorsque la HAS estime que la prise en charge par l'Assurance maladie est justifiée, mais que des données complémentaires sont nécessaires, elle peut conditionner le renouvellement de la prise en charge par la réalisation par les industriels concernés d'une étude post-inscription.

L'ensemble de ces programmes et dispositions réglementaires devrait par conséquent permettre d'assurer un continuum pour l'innovation, pour autant que leur application devienne effective, comme pour le forfait innovation par exemple (figure 2).

Les propositions consensuelles de la table ronde sont les suivantes :

- garder la cohérence, quel que soit le programme ministériel, avec le plan de développement clinique international ;

- mettre en œuvre effectivement et rapidement le forfait innovation (art. 165-1-1) ; ${ }^{[3]}$

- fluidifier et intégrer les procédures.

Enfin, sur le sujet précis des techniques innovantes, une divergence a vu le jour entre les membres de la table ronde et n'a pas pu être résolue. En effet, il a été proposé de mettre en place une procédure de codage précoce des technologies innovantes avec l'idée de répondre au constat suivant : un dispositif médical ayant obtenu son marquage CE peut être utilisé en France dans un établissement de santé (il s'agit bien ici d'un dispositif médical innovant dont la catégorie n'aurait pas encore été repérée et possiblement inscrite sur la liste des catégories intra-GHS à évaluer par la HAS selon l'article 165-11 du CSS). ${ }^{[2]}$

S'agissant d'une technologie innovante, l'acte associé n'existe pas. En réalité, ces technologies innovantes et non encore validées sont utilisées via des codages d'actes et de GHS inadaptés avec un 


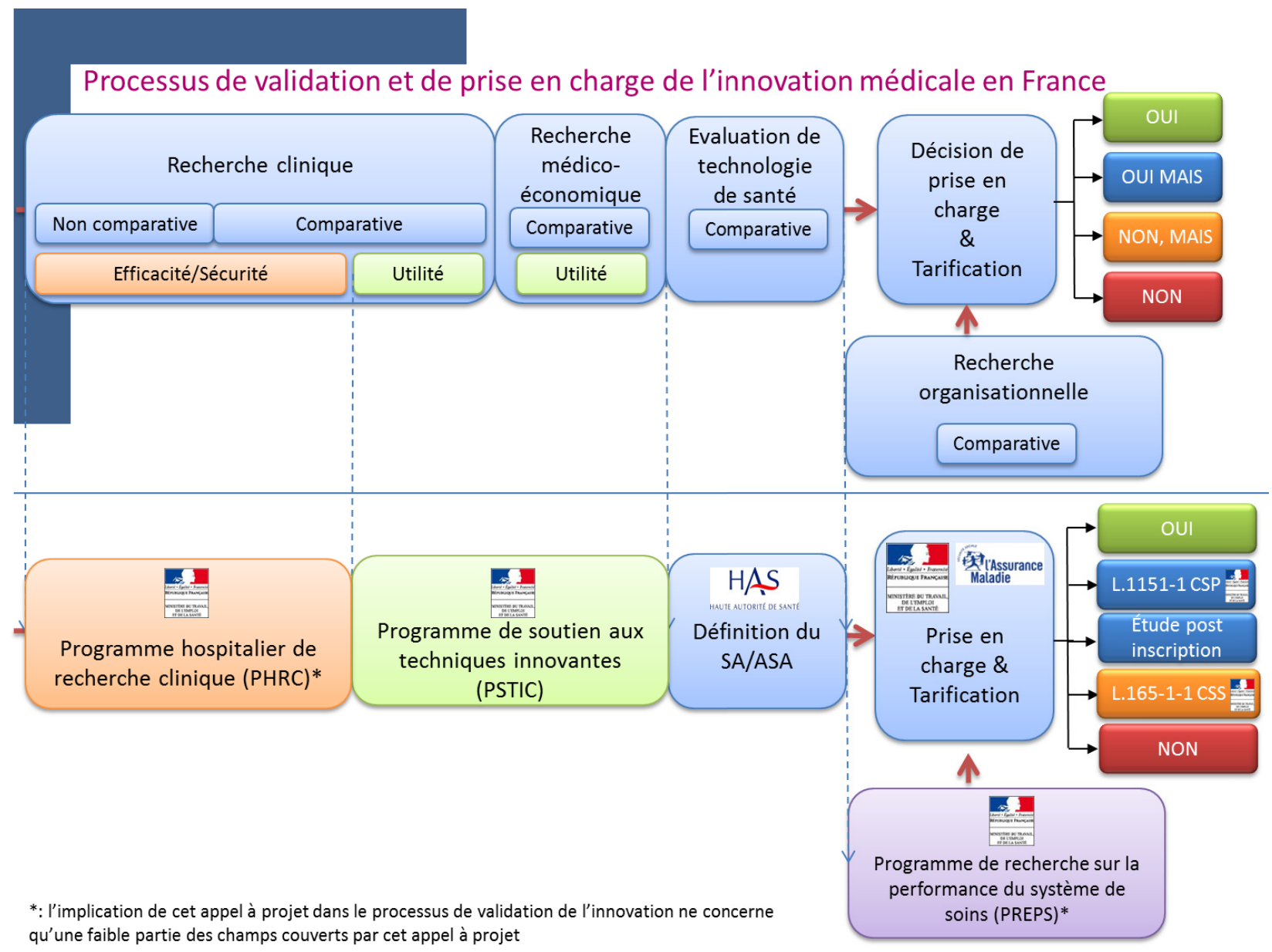

\section{Source : DGOS-PF4}

Fig. 2. Continuum de l'innovation en France selon programmes de la DGOS. Avec l'aimable autorisation du Dr Carbonneil.

On note que la recherche médico-économique est ici placée en amont de l'évaluation par la HAS par association avec le programme STIC. Néanmoins, la mise en application à partir d'octobre 2013 du décret 2012-1116 du 2 octobre 2012 relatif aux missions médico- économiques de la Haute Autorité de santé permettra de clarifier le type de données économiques requises pour la prise en charge des dispositifs médicaux en dehors des STIC.

DGOS : Direction générale de l'offre de soins ; HAS : Haute autorité de santé ; SAASA : service attendu/amélioration du service attendu ; STIC : soutien aux techniques innovantes et coûteuses.

financement partiel et imparfait qui repose sur le budget propre de l'établissement de santé ou un financement de l'industriel. Cette utilisation n'est pas systématiquement réalisée dans le cadre d'études cliniques, avec la crainte que la courbe d'apprentissage nécessaire au bon usage de la technologie ne soit pas au niveau requis.

La problématique soulevée concerne finalement plus le suivi de l'innovation que son financement à proprement parler, puisque sa validation n'étant pas définitive, la pertinence de sa prise en charge par la collectivité pose encore question. L'objectif de ce codage précoce, très probablement à centrer sur l'acte, serait de repérer l'usage qui est fait de cette technologie innovante et des pratiques médicales, de cerner les besoins du patient et si possible de mesurer son impact à partir des bases de données et des nomenclatures existantes. Il faudrait toutefois que ce codage soit à la fois bien compris et effectivement réalisé en pratique par les professionnels de santé, bien qu'il ne soit pas associé forcément à un financement.

Ce sujet est difficile car il pourrait remettre en cause, du moins partiellement, l'accès libre à l'intra-GHS. De plus, il peut sembler paradoxal de présenter une disposition de codage précoce sans financement temporaire systématisé comme une solution d'accompagnement de l'innovation.

Néanmoins, la lisibilité de l'usage fait en France d'une innovation va participer à imposer la réflexion sur l'efficacité, l'utilité et la prise en charge future. 
De plus, la mise en place progressive des nouvelles dispositions telles que l'évaluation par la HAS de certaines catégories de dispositifs intra-GHS pourra éclairer sur les solutions envisageables en pratique, sans cerner toutefois la globalité de la problématique puisque l'acte n'est pas concerné par cette mesure.

Il n'a pas été possible à ce stade de nos réflexions d'avancer plus loin sur ce point complexe et sensible qui mériterait à lui seul une table ronde dédiée.

\section{Limites et perspectives}

Les limites des travaux de cette table ronde reposent sur un défaut de représentativité de certains acteurs tels que les médecins libéraux et les PME. De plus, même si certains éléments sont bien évidemment communs, les sujets du handicap et de la télémédecine auraient mérités d'être abordés, mais cela n'a pu être fait par manque de temps.

De plus, une réflexion concernant les évolutions du corpus de données nécessaire pour accéder au financement dans le dispositif médical doit être réalisée : critères de jugement, internationalisation des données, données requises pour les biomarqueurs compagnons, données économiques. En effet, les propositions de la table ronde, qu'elles soient consensuelles ou en discussion, ne sauraient être comprises comme un palliatif à une insuffisance de données cliniques pertinentes dans le dossier de demande d'évaluation pour prise en charge des actes et des dispositifs médicaux.

\section{Conclusion}

Les travaux de la table ronde ont permis d'analyser en profondeur les procédures d'évaluation et de tarification des dispositifs médicaux et des actes associés. Les points d'amélioration ont ainsi pu être mis en lumière et des propositions concrètes formulées.

Enfin, les points de discussion, très ouverts, permettent d'engager des pistes de réflexion en vue d'aboutir à des procédures plus efficientes pour le système de santé français et les patients pris en charge.

Remerciements. Nous remercions vivement l'ensemble des participants à la table ronde $\mathrm{N}^{\circ} 1$ des XXVIII ${ }^{\mathrm{es}}$ Ateliers de Giens.

\section{Conflits d'intérêts. Aucun.}

Abréviations. CCAM : classification commune des actes médicaux ; CEPS : Comité économique des produits de santé ; CNEDiMTS : Commission nationale d'évaluation des dispositifs médicaux et des technologies de santé ; CSP : Code de la santé publique ; CSS : Code de la sécurité sociale ; DGOS : Direction générale de l'offre de soins; ES : établissement de santé;
ENCC : étude nationale des coûts à méthodologie commune ; FFR : flux coronaire résiduel (fractional flow reserve); GHM : groupe homogène de malades ; GHS : groupe homogène de séjour ; HAS : Haute autorité de santé ; HPST : (loi) «Hôpital, patients, santé, territoire »; INCa : Institut national du cancer ; LPP : liste des produits et prestations remboursables; NABM : nomenclature des actes de biologie médicale ; NGAP : nomenclature générale des actes professionnels ; PHRC : programme hospitalier de recherche clinique ; PSTIC : programme de soutien aux techniques innovantes et coûteuses ; T2A : tarification à l'activité ; UNCAM : Union nationale des caisses d'Assurance maladie.

\section{Annexe 1}

Pour être éligible à la liste des produits et prestations remboursables (LPP), le dispositif doit être à usage individuel et pouvoir être inscrit sur l'un des titres suivants:

- Titre I : Matériels et traitements à domicile, aliments diététiques, articles pour pansements

- Titre II : Orthèses et prothèses externes

- Titre III : Dispositifs médicaux implantables

- Titre IV : Véhicules pour personnes handicapées physiques

\section{Annexe 2}

Le Comité économique des produits de santé est un organisme interministériel placé sous l'autorité conjointe des ministres chargés de la santé, de la sécurité sociale et de l'économie. Il propose les tarifs de remboursement des médicaments et des dispositifs médicaux à usage individuel et des prestations de service pris en charge par l'Assurance maladie et réalise le suivi des dépenses afférentes.

Le Conseil de l'hospitalisation a pour rôle d'émettre des recommandations à l'intention du Ministre concernant l'élaboration de la politique de financement des établissements de santé et la détermination des objectifs de dépenses d'assurance maladie relatives au frais d'hospitalisation.

\section{Références}

1. Article L165-1 du Code de la sécurité sociale. http://www.legifrance.gouv.fr

2. Article L165-11 du Code de la sécurité sociale. http://www.legifrance.gouv.fr

3. Article L165-1-1 du Code de la sécurité sociale. http://www.legifrance.gouv.fr

4. Article L1151-1 du Code de la santé publique. http://www.legifrance.gouv.fr

Correspondance et offprints : Frédérique Debroucker, Medtronic France SAS, 27 quai Alphonse le Gallo, CS 30001, 92513 Boulogne-Billancourt Cedex, France.

E-mail : frederique.debroucker@medtronic.com 